Gynäkol Geburtschilfliche Rundsch 1995;35:158

\title{
Brief an die Schriftleitung
}

Der Unterzeichnete ersucht um Publikation eines Korrigendums

einer Passage in seinem Buch

«Die Wertheim-Klinik», Wien, Maudrich, 1992,

im Hinblick auf

Die Bildtafeln in der Wertheim-Monographie von 1911

Sachverhalt, Berichtigung und Entschuldigung an Herrn Prof. Zander

Die von Ernst Wertheim verfasste und 1911 im Verlag Urban \& Schwarzenberg erschienene Monographie «Die erweíterte abdomi-nale Operation bei Carcinoma colli uteri (auf Grund von 500 Fallen)* enthält bei einem Gesamtumfang von 223 Seiten zwischen den Seiten 166 und 167 im Exemplar der Österreichischen Nationalbi-bliothek (488 935 C) und im Exemplar des Institutes für Geschichte der Medizin an der Universität Wien (46 679) 6 nicht paginierte, die Operationstechnik illustrierende Bildtafeln; im Exemplar der Uni-versitätsbibliothek Wien (II350 617) sind diese Bildtafeln am Schluss des Buches, somit nach Seite 223, gleichfalls nicht paginiert, eingeheftet. In dem von Ernst Wertheim der II. Universitätsfrauen-klinik Wien handschriftlich zugeeigneten Exemplar (771) fehlen die Bildtafeln.

Die auf dem letzteren und somit zweifelsfrei unvollständigen Monographieexemplar basierende, die Abbildung im Wertheim-Buch betreffende Darstellung in der 1992 im Verlag Wilhelm Maudrich erschienenen Monographie «Die Wertheim-Klinik, eine Geschichte der II. UniversitätsFrauenklinik Wien > > ist demzufolge unrichtig. Richtig ist, dass Wertheim seine 1911 erschienene Monographie mit Bildtafeln, die von I bis VI durchnumeriert sind, illu-strierte. Josef Zander [1] ist folgerichtig in seinem Beitrag «Zur Geschichte der Gynäkologie und Geburtshilfe» kein Irrtum unterlaufen, als er in diesem 2 von den insgesamt 6 die Operationstechnik illustrieren-den Originalabbildungen aus der Wertheim-Monographie von 1911 wiedergab.

Der Autor der medikohistorischen Monographie «Die Wertheim-Klinik, eine Geschichte der II. Universitäts-Frauenklinik Wien» bittet

Herrn o. Univ.-Prof. emeritus Dr. Dr. h.c. Josef Zander

in aller Form um Entschuldigung für den ihm unterlaufenen Fehler.

Anton Schaller

1 Zander J: Meilensteine in der Gynäkologie und Geburtshilfe - 100 Jahre Deutsche Gesellschaft für Gynäkologie und Geburtshilfe; in Beck L (Hrsg): Zur Geschichte der Gynäkologie und Geburtshilfe. Berlin, Springer, 1986, p41.

158 